\title{
'Imaka: a one-degree high-resolution imager for the Canada-France-Hawaii Telescope
}

\author{
Mark R. Chun ${ }^{*} a$, \\ Raymond G. Carlberg ${ }^{b}$, Harvey B. Richer ${ }^{c}$, Yannick Mellier ${ }^{d}$, Pierre Astier ${ }^{e}$, \\ Olivier Lai, Derrick A. Salmon, Jean-Charles Cuillandref, \\ David Andersen, John Pazder, Jean-Pierre Véran ${ }^{g}$, \\ Gregory A. Barrick, Steven Bauman, Kevin K. $\mathrm{Ho}^{f}$, \\ Remy Avila ${ }^{h}$,Richard W. Wilson, Timothy Butterley ${ }^{i}$ \\ 'Imaka science team \\ ${ }^{a}$ University of Hawaii (USA); ${ }^{b}$ Univ. of Toronto (Canada); ${ }^{c}$ The Univ. of British Columbia \\ (Canada); ${ }^{d}$ Institut d'Astrophysique de Paris (France); ${ }^{e} \mathrm{IN} 2 \mathrm{P} 3$ (France) \\ ${ }^{f}$ Canada-France-Hawaii Telescope (USA); ${ }^{g}$ National Research Council Canada (Canada); \\ ${ }^{h}$ Univ. Nacional Autónoma de México (Mexico); ${ }^{i}$ Durham Univ. (United Kingdom)
}

\begin{abstract}
The 'Imaka project is a high-resolution wide-field imager proposed for the Canada-France-Hawaii telescope (CFHT) on Mauna Kea. 'Imaka takes advantage of two features of the optical turbulence above Mauna Kea: weak optical turbulence in the free-atmosphere and boundary layer turbulence which is highly confined within a surface layer tens of meters thick and or the telescope enclosures. The combination of the two allows a groundlayer adaptive optics system (GLAO) to routinely deliver an extremely-wide corrected field of view of one-degree at an excellent free-atmosphere seeing limit at visible wavelengths. In addition, populating the focal-plane with orthogonal-transfer CCDs provides a second level of image improvement on the free-atmosphere seeing and the residual GLAO correction. The impact of such an instrument covers a broad range of science and is a natural progression of CFHT's wide-field expertise.
\end{abstract}

Keywords: adaptive optics, wide-field imaging, ground-layer

\section{INTRODUCTION}

The Canada-France-Hawaii Telescope (CFHT) is located at one of the premier sites in the world for groundbased astronomy and while the superb seeing on Mauna Kea has long been known, a delivered image quality commensurate with the site has yet to be fully realized even at the most modern facilities on the summit. Numerous optical turbulence studies ${ }^{1-3}$ at Mauna Kea have found excellent free-atmosphere seeing above the site and recent studies ${ }^{3}$ find that the turbulence within the boundary layer (e.g. first kilometer) is confined to just the first tens of meters. This ground layer is roughly equal in strength to the free-atmosphere seeing so nearly half of the optical turbulence arises within a few tens of meters of telescope. In addition, studies at $\mathrm{CFHT}^{4}$ have found that the dome seeing is particularly strong. A facility that is able to negate this local seeing would produce unprecedented resolutions over these large fields of view. 'Imaka is a path to achieve this on CFHT.

Aptly named after the Hawaiian word $\bar{i} m a k a$ meaning scenic viewpoint", 'Imaka deploys a combination of technologies to remove the local optical turbulence: a ground-layer adaptive optics system, a focal-plane

\footnotetext{
*MC: University of Hawaii, Institute for Astronomy, 640 N. Aohoku Place, Hilo, HI, USA; mchun@ifa.hawaii.edu

${ }^{*}$ http://www.wehewehe.org
} 
consisting of orthogonal-transfer CCDs (OTCCD), and a new set of optics capable of delivering high-resolution images. In addition, 'Imaka will benefit greatly from the gains made by CFHT's current efforts to vent the telescope enclosure. This mixture of active and passive approaches to mitigate the local degradations will deliver wide corrected fields of view, angular resolutions 2-3 times better than the current CFHT/MegaCam widefield imager, and point-source sensitivities comparable to that of current 8-10 meter class telescopes. Optical turbulence measurements at the site along with simulations of the performance of a ground-layer adaptive optics system with an OTCCD focal plane indicate that 'Imaka will deliver angular resolutions of $F W H M \sim 0.3^{\prime}$ at visible wavelengths (e.g. r-band/0.7 microns) over a one-degree field of view. The delivered image quality varies with wavelength with better performance at redder wavelengths and worse performance at bluer wavelengths. Notably, the angular resolutions are within a factor of 2-3 of that of the Hubble Space Telescope but with a field of view nearly 400 times larger than ACS/WFC3. The potential is striking and the scientific impact covers a broad range of areas from the Solar System to the most distant objects in the universe.

In this proceeding we present the conclusions of the 'Imaka feasibility study we made for CFHT. We present the science impact and examples from the science case as well as resolutions of the key technical feasibility questions: (1) Is an opto-mechanical design for the instrument that meets the basic instrument performance and functional requirements feasible?, (2) Can we confirm and quantify that the image quality at CFHT is dominated by local seeing and that the free-atmosphere seeing is low?, and (3) Do we understand the key error terms for a GLAO system with this extreme field size and wavelength range?. More information on the study can be found in contributions in these proceedings by Lai et al. (performance simulations), Evans et al. (optical design), and the study report (http://www.cfht.hawaii.edu/en/projects/IMAKA/).

\section{SCIENCE CASE}

'Imaka is a general-purpose imager with an unmatched combination of angular resolution and field. While it draws on techniques and technologies developed for advanced adaptive optics systems, 'Imaka's field and angular resolution link its science to that of wide-field imaging and survey facilities. The science cases remain true to CFHT's current scientific legacy in wide-field imaging spanning the Solar system, stellar physics, galactic structure, galaxy evolution, and cosmology. 'Imaka's unique combination of high angular resolution and field of view will provide a new avenue for discovery.

As a concrete illustration of the gain brought by 'Imaka, we compare the current CFHT/MegaCam imager to CFHT with 'Imaka. Taking into account the expected resolutions 'Imaka will obtain (see Section 5), the gain in quantum efficiency at longer wavelengths from more modern CCD devices, and the expected optical throughputs of the 'Imaka, we find that there is a sensitivity gain of between 0.7-1.8 magnitudes from $g$-band (0.45 microns) to $z$-band (0.9 microns) of 'Imaka over the current MegaCam respectively. In addition, if 'Imaka deploys redsenstitive CCDs, the $Y$-band (1.0 microns) photometric window is opened but with a loss of the $u$-band window with respect to MegaCam. The comparison can also be expressed in terms of the speed at which 'Imaka can cover the sky to a given depth ("survey speed"). The roughly 1000-hours spent observing the 4-square degree CFHT Legacy Survey Deep fields in $g, r, i$, and $z$ (with depths of about $r_{A B} \sim 28.4$ ) could be done in nearly an order of magnitude less time with 'Imaka. While impressive, 'Imaka must be considered within the context of more modern facilities that will be functional in the coming years.

Wide-field optical imaging is truly a burgeoning domain with many high profile facilities expected to come online over the next decade. In some cases these facilities are competitive capabilities (e.g. Subaru/HyperSuprimeCam, PanStarrs4, and LSST) while others are complementary (e.g. TMT, JWST, EUCLID). It is instructive to quantify this comparison. The typical wide-field imager metric of etendue $=A \Omega$, expressing how fast a facility can cover area, does not account for 'Imaka's gain in angular resolution. Indeed, etendue does not distinguish 'Imaka from CFHT's current MegaCam. On the other hand, the Strehl ratio, a typical performance metric used for adaptive optics systems, does not account for 'Imaka's four orders of magnitude gain in field of view over classical AO systems. In fact, the Strehl of 'Imaka is essentially zero given the relatively large residual wavefront phase errors from the free-atmosphere.

We adopt a slight evolution of etendue to a more tangible metric to evaluate the relative performance of facilities covering large areas of sky with good image quality. Here we use a metric that quantifies the rate at 
which information, namely the signal-to-noise ratio for background-limited point sources, is collected over the field of view. We express this as:

$$
F O M=\frac{\mathbf{A} \Omega}{\mathbf{I Q}^{2}}
$$

where $A$ is the collecting area of the telescope, $\Omega$ is the field of view, and $I Q$ is the delivered image quality on the focal plane.

Table 1 shows the growth of this figure of merit for current and future ground-based optical wide-field imagers. 'Imaka's competition comes from the significantly larger projects such as Subaru/HyperSuprimeCam and LSST. Here the telescope apertures and field sizes beat 'Imaka in terms of collecting signal on sources over large fields. We note also that, while having a very different science objective, the Gemini multi-conjugate adaptive optics system $\left(\mathrm{GEMS}^{5}\right)$ with its 80-arcsecond diameter field of view and diffraction-limit angular resolutions in $\mathrm{H}$-band (1.65 microns) has a normalized figure of merit of 0.5. Similarly, a multi-object adaptive optics (MOAO) system, while providing a diffraction-limited image does so for very small fields (discrete objects) within a larger field and thus has a very small figure of merit. It is clear, however, that the scientific objective of these systems varies drastically from the 'Imaka case.

Table 1. A comparison of a variety of existing or planned ground-based wide-field imaging facilities for the $F O M=\frac{A \Omega}{I Q^{2}}$. In the table the quantity $D_{\text {eff }}$ is the square root of the collecting area of the telescope and DIQ is the delivered image quality at the facility. Note that the figure of merit is normalized by that for CFHT/MegaCam.

\begin{tabular}{|l|c|c|c|c|c|c|}
\hline Instrument & Year & Site & $\begin{array}{c}D_{\text {eff }} \\
(\text { meters })\end{array}$ & $\begin{array}{c}\text { FOV } \\
\left(\text { degrees }^{2}\right)\end{array}$ & $\begin{array}{c}\text { DIQ } \\
(\text { arcsec }\end{array}$ & $\begin{array}{c}\text { Metric } \\
\text { (normalized) }\end{array}$ \\
\hline PS1 & 2009 & Haleakala & 1.6 & 7.30 & 1.0 & 0.9 \\
\hline MegaCam & 2003 & Mauna Kea & 3.5 & 1.0 & 0.75 & 1.0 \\
\hline ODI & 2012 & Kitt Peak & 3.3 & 1.0 & 0.60 & 1.4 \\
\hline SuprimeCam & 2002 & Mauna Kea & 8.1 & 0.25 & 0.65 & 1.8 \\
\hline PS2 & 2012 & Haleakala & 1.6 & 7.30 & 0.7 & 1.8 \\
\hline DEC & 2012 & Cerro Tololo & 3.9 & 3.0 & 0.95 & 2.3 \\
\hline 'Imaka & 2016 & Mauna Kea & 3.5 & 0.8 & 0.3 & 5.0 \\
\hline HyperSuprimeCam & 2012 & Mauna Kea & 8.0 & 1.5 & 0.65 & 10.4 \\
\hline PS4 & $? ? ? ?$ & Mauna Kea & 3.1 & 7.3 & 0.55 & 10.6 \\
\hline LSST & 2016 & Cerro Pachon & 6.7 & 9.6 & 0.7 & 40.4 \\
\hline
\end{tabular}

Even with the improved metric, Table 1 does not take into full consideration the fact that 'Imaka will deliver an angular resolution unique to all the ground-based wide-field imaging facilities in the coming decade. Here a comparison with space-based missions may be more appropriate. In particular, we call out the NASA/JDEM and ESA/EUCLID missions. Both projects are designed for weak lensing and are expected to provide stable high quality images with wide-field optical imagers on 1-2 meter class telescopes. It is not clear when JDEM will be operational but EUCLID is expected to fly in 2019. However, in its present design Euclid has only one very broad visible filter $(\mathrm{r}+\mathrm{i}+\mathrm{z})$ for ellipticity measurements and will rely on multi-band wide-field surveys in the visible from the ground. Therefore 0.3 -arcsecond wide-field visible surveys with 'Imaka would turn out to be very useful for Euclid, in particular for the the g and r-band data. Until 2019, 'Imaka will therefore outperform any other wide-field instrument on the image quality front but will still be valuable for ground based follow up later on.

'Imaka's uniqueness is in the combination of field and resolution. In addition, operationally many of these facilities will be dedicated to large surveys. Access for P.I. driven programs will be limited. Here CFHT's community's experience in wide-field imaging will be a key benefit to tap the capabilities of 'Imaka.

\subsection{Science Case Examples}

As a concrete example of the unique science that 'Imaka can enable, we present one of the science cases from the 'Imaka Feasibility study (http://www.cfht.hawaii.edu/en/projects/IMAKA/): Resolved stellar populations in 
nearby galaxies out to the Virgo cluster. For this case, the angular resolution allows us to resolve the individual stellar populations within the galaxies, the multi-band wavelength coverage provides the spectral information to characterize the populations, and the field coverage allows a complete census of the galaxy and it's environment to be made without worries of selection effects or the mis-choice of unrepresentative fields within the galaxy.

Many of the key predictions of galaxy formation theories - such as the spatial distribution and morphology of the structure and substructures in the outer regions of galaxies - are only testable for the nearby Universe. Thus observations of the stellar populations of these galaxies provide direct tests of and constraints on cosmological models of galaxy formation. New surveys in both the Milky Way (e.g. SDSS) and M31 (e.g. PAndAS) have recently uncovered a spectacular panorama of the complex outer regions of galaxies, with a plethora of dwarf galaxies, stellar streams and diffuse structures. The luminosity function of these dwarf galaxies and the expectations from simulations of galaxy formation indicate that many more lower luminosity dwarfs exist. 'Imaka offers a wide field of view with increased sensitivity, ideally suited to finding these compact objects in a large stellar halo such as M31. A more complete census of dwarf galaxies directly confronts hierarchical cosmological models on small scales. The sharper PSF will also allow the giant and horizontal branch populations of these galaxies to be traced into the core, and therefore lead to a much more detailed analysis of the structural properties and radial gradients of these systems. Such data, even for the currently discovered satellites, will aid in our interpretation of the causes of the different properties of the satellite system (both internally and when comparing Milky Way dwarfs to M31 dwarfs). For example, it is still unclear whether the observed differences are caused by in situ processes within the dwarf satellites or through dynamical interactions with the host.

Pushing to more distant galaxies in the local volume - where there is a statistically larger sample of galaxies over a full range of morphological type - requires wide field capabilities with high spatial resolution to overcome crowding issues, and demonstrates the need for wide-field AO capabilities on large telescopes that approach the diffraction limit. 'Imaka will extend the science which is currently only possible in the Local Group to all galaxies within $10 \mathrm{Mpc}$. At $10 \mathrm{Mpc}$, the 1 square degree field corresponds to $175 \mathrm{kpc}$. Such data would allow one to measure the spatial structure of extra-planar stars, measure axial ratios, map halo flattening to very faint isophotes, determine the metallicity distribution of halo stars and their spatial variation, search for substructures or clumpiness due to tidal streams or dissolving dwarf galaxies, measure structural parameters of globular clusters and find asymptotic giant branch (AGB) stars associated with intermediate-age stellar populations. A hint of things to come can be seen in the recent paper by Mouhcine et al $(2010)^{6}$ where spectacular unexpected structure (a giant stream, a large flat cocoon-like structure out to $40 \mathrm{kpc}$ from the galaxy) was discovered surrounding NGC 891 from Subaru imaging. This galaxy is located at $10 \mathrm{Mpc}$ from the Milky Way and records details of halo structure formation at distances well outside the Local Group. Observations of this sort will answer questions such as did galaxies accrete most of their mass/stars at early or late times? How many mergers does a typical galaxy undergo? What is the lowest mass dwarf galaxy which can form stars? Currently,these questions are restricted to the Milky Way, M31 and M33. 'Imaka will be able to probe the outer haloes of these latter galaxies to typically $35 \mathrm{mag} / \mathrm{sq}$. arcsec - at least one magnitude deeper than current CFHT/MegaCam studies. Obtaining a significant sample of resolved stellar halos to low surface brightness is central to advancing cosmological models of galaxy formation. 'Imaka will provide a new perspective on our nearest neighbors. For this work, it will be necessary to measure at least the top 1 magnitude of the RGB out to $10 \mathrm{Mpc}$. At that distance the RGB tip occurs at $\mathrm{i}=25.8$, so we require data that reach $\mathrm{i}=26.8, \mathrm{~g}=28$ with $S / N \sim 10$.

Current HST-based projects are probing some nearby galaxies with these science goals, but all are placed at a serious disadvantage due to the impossibility of observing a large fraction of the area of nearby galaxies with the (relatively) small field of ACS/WFC3. High resolution wide field cameras such as 'Imaka are an essential prerequisite for this science. A lesson learned from these surveys is that no firm conclusions regarding the properties of the outskirts of galaxies can be drawn from observations sampling unrepresentative galactic volumes. An eloquent example of this is the single pointing ultra-deep ACS observations of a minor-axis field in the halo of M31 that was realized later to fall close to a highly disturbed and clumpy structure discovered in the panoramic survey of M31. Panoramic coverage provides the only avenue to unlock the secrets of the outskirts of galaxies, and to fully exploit what they have to tell us about galaxy assembly. 


\section{INSTRUMENT CONCEPT}

The instrument concept for 'Imaka has changed significantly from that presented in Lai et al. ${ }^{7}$ The basic performance and functional requirements, nonetheless, have stayed largely unchanged.

\section{Top-Level Performance Requirements}

1. Field of view : 1 degree diameter or equivalent solid angle

2. Wavelength Range : 0.4 to 1.1 micron

3. Delivered image quality of 0.3 " or better at r-band under median seeing conditions with a FWHM uniform within $10 \%$ over entire field

4. Photometric measurements with an accuracy of $1 \%$ absolute and $0.1 \%$ relative

5. Astrometric measurements with an accuracy of 40mas absolute and 0.8 mas relative

6. Sky coverage $100 \%$ in Galactic plane and $>50 \%$ at North Galactic Pole

\section{Top-Level Functional Requirements}

1. A GLAO system with (1) wavefront sensors that sense the wavefront aberrations that arise from effects near the ground and (2) a DM that corrects this wavefront across the field.

2. A focal-plane camera providing local tip/tilt wavefront corrections

3. 'Imaka can not preclude the use of other CFHT facility instruments

4. Access to the CCD camera and DM systems should be provided with 'Imaka on the telescope.

5. An exchange to 'Imaka should be completed in less than one working day

The instrument concept has evolved into two possible configurations: 'Imaka-Prime a two-channel prime focus design and 'Imaka-Cassegrain: a double-pass transmissive design at the Cassegrain focus. Given the large field size and high image quality, both designs are large in scale and will need either a dedicated upper end or full access to the volume behind the primary mirror cell. In addition to the imaging optics, we require that the CFHT telescope be kept in roughly its current operational configuration. In particular, it must be compatible with existing or currently planned instrumentation. A new telescope top end is consistent with this requirement though changes to the cassegrain focus optical prescription is likely not. To support the GLAO correction, we also require that the instrument have (1) a well defined and accessible intermediate telescope pupil where an deformable mirror (DM) can be placed and (2) a set of approximately 6-8 wavefront sensors that can be deployed about the field. To support the additional tip/tilt correction in the free-atmosphere, we require a CCD camera system based on orthogonal transfer (OT) technology with a broad wavelength coverage. Given the desire to have use wide bandpasses for the science and the sharp image quality, 'Imaka will also require an atmospheric dispersion corrector. Finally, as with previous instrumentation at CFHT, 'Imaka will need an instrument calibration and data reduction pipeline to provide astronomy-ready images with instrumental signatures removed.

With the limited resources for the feasibility study we concentrated on those issues that pose the largest questions for its feasibility. Other, better understood issues, where technologies or current practices seem to be better defined are left to Phase A engineering studies where more detailed design and systematic decisions will start to be made. We therefore left details of the ADC, DM and control, wavefront sensors and the data pipeline in this latter category. From a technical stand point we identified the overall optical design, the insitu turbulence measurements at CFHT, and quantifying the major GLAO error terms as the critical technical questions to address in the feasibility study. In this section we present the two optical designs and in the two sections that follow we present the turbulence measurements and simulation results. 


\subsection{Optics}

The biggest challenge in the optical design was to determine, given the field size, whether or not a design was even feasible. The initial concept using a deformable secondary mirror was discarded once the impact on the delivered performance of the misconjugation of the secondary mirror $\left(h_{D M} \sim-43\right.$ meters $)$ was quantified. The top level instrument requirements flow from the 'Imaka science cases and functional limitations imposed by the telescope. Given these requirements, the optical designers were faced with the following challenges and constraints:

- Provide images with $80 \%$ encircled energy diameters 0.15 arcsec (or better) across a field 1 degree in diameter (or equivalent) for each spectral band (e.g. g,r,i,z,Y)

- Deliver a final f/5.7-f/6.8 optical beam to match the desired image sampling of 0.1 arcsec per pixel for an orthogonal transfer CCD with a 10-12 micron pixel pitch.

- Provide access at a well defined pupil image to locate an adaptive optical element (DM) working at an incident angle of less than 20 degrees and conjugate to a zone between $15 \mathrm{~m}$ below the primary mirror to $30 \mathrm{~m}$ above the primary mirror (see Lai et al. 2010 these proceedings).

- Locate optical and CCD camera components at accessible locations on the telescope

- If at the Cassegrain focus, pass the beam through the primary mirrors central hole.

- Use refractive materials (if any) that are available commercially at the required sizes.

The optical design requirements pose a serious design challenge. The needs for a large focal surface ( 360 $\mathrm{mm}$ diameter), and for an adaptive element near a pupil image, requires 'Imaka to be physically large. A further constraint of the large focal surface is that in order to feed light to an array of wavefront sensors with minimal vignette of the science focal plane we will need to place wavefront sensor pickoff mirrors very close to the final focal surface. As a result, the wavefront pickoffs may be located behind the optical bandpass filters placing constraints on guide star availability.

Because of the difficulty inherent to the 'Imaka optical design problem we engaged the services of 6 optical designers familiar with designing high performance optical systems for astronomical facilities over sixteen design variants. Of these, the following two concepts ('Imaka-Prime and 'Imaka-Cassegrain) meet the core requirements of image quality, field size, pupil image quality, conjugation and focal surface access.

\subsubsection{Prime Focus Design}

The prime focus design by Clinton Evans and Hua Lin (Evans et al. 2010 these proceedings) splits the field into two rectangles measuring 0.4 degrees x 1.0 degree with centers separated by 2 degrees on the sky. Two separate and independent optical systems together providing areal sky coverage equivalent to a one degree diameter field, are mounted outboard of the telescope top ring.

The all-reflective design, one channel of which is illustrated on the left in Figure 1, uses 2 flat mirrors, 2 aspheres and a mildly convex spherical DM $260 \mathrm{~mm}$ in diameter. The two channels mount to a dedicated upper end. The aspheres are large, $65 \mathrm{~cm}$ and $90 \mathrm{~cm}$ respectively, which will make fabrication and testing expensive. In addition, the focal surface is curved $(\mathrm{r}=1.6 \mathrm{~m})$ but can accommodate flat $4 \mathrm{k} \times$ x $4 \mathrm{k}$ OTCCDs without serious IQ degradation if they are offset in focus in steps of $60 \mathrm{um}$. As currently laid out, each unit will vignette a small portion of the others pupil as well as an even smaller portion of its own pupil. The effects are small and amount to an surface area loss of less than the current CFHT/MegaCam configuration.

The key advantages of this design are: (1) its reflective design is the achromatic, (2) it minimizes of the number of surfaces that can give rise to ghosts, and (3) its two-channel approach has inherent redundancy. The key disadvantages are the large aspheric optics and the need for two largely independent GLAO systems.

A more in-depth discussion of the Prime focus design and its performance can be found in Evans et al (2010, these proceedings). 

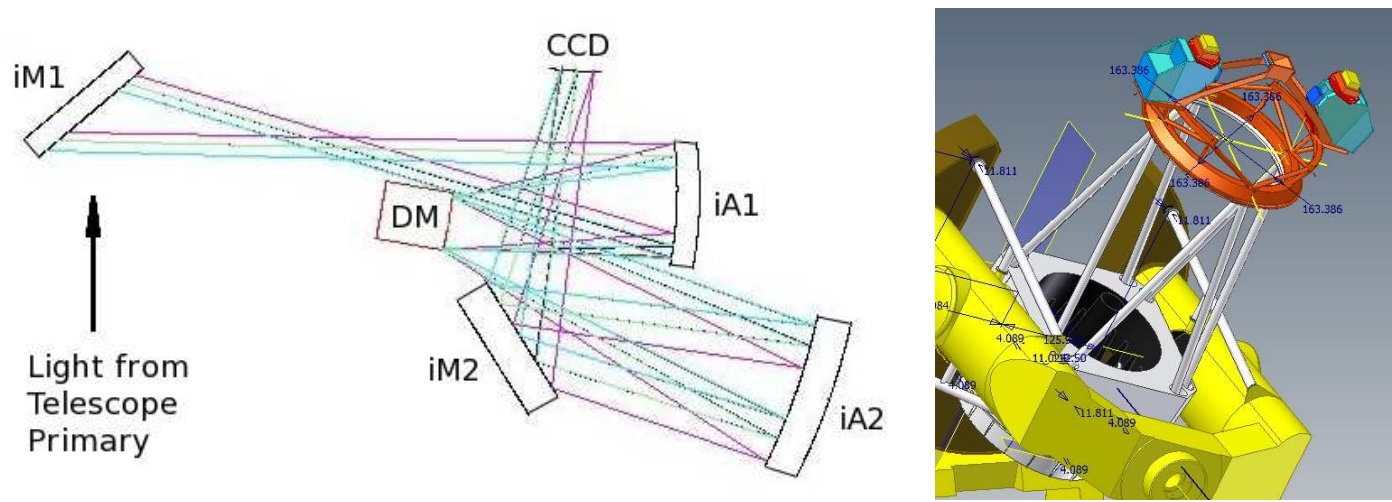

Figure 1. Left: One side of the 'Imaka prime focus design (Evans et al. 2010 these proceedings). The light coming from the CFHT primary mirror is reflected of a flat secondary mirror (iM1) and sent towards the outer ring of the telescope. There two aspheres (iA1, iA2), the deformable mirror (DM), and a last fold mirror (iM2) bring the light to the science focal plane (CCD). Not shown are the wavefront sensor or ADC optics. Right: A 3D CAD rendering of the 'Imaka-Prime layout showing the two channels at the top of the CFHT 3.6-m telescope.
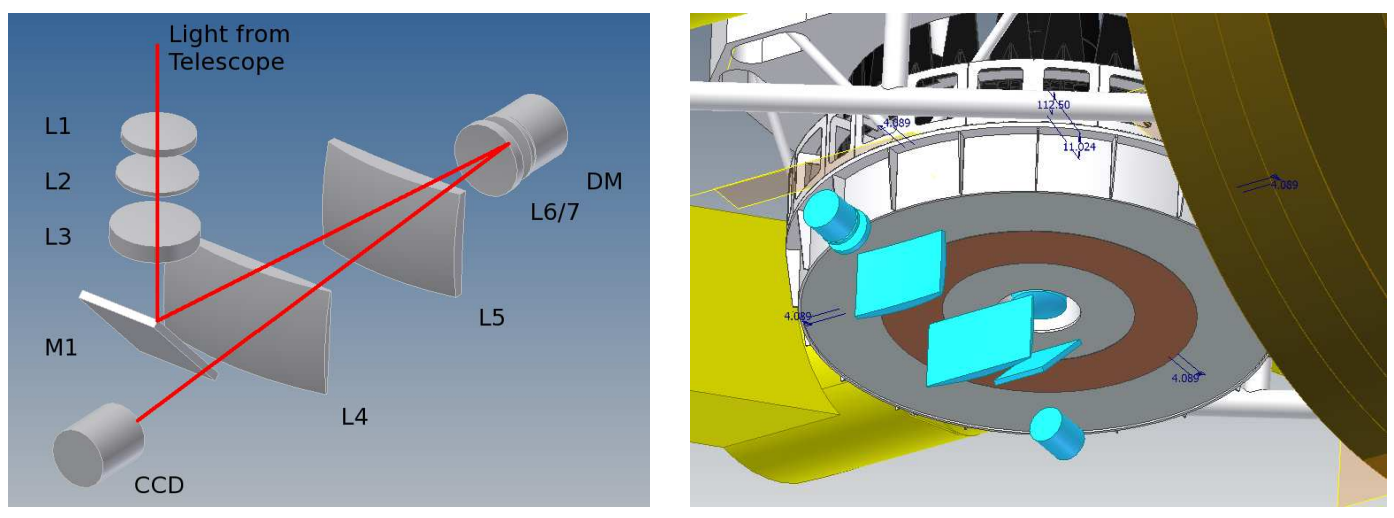

Figure 2. Left: The optical path of the 'Imaka Cassegrain focus design by John Pazder (HIA). The light coming from the CFHT f/10 focus is sent through a three-element system (L1-L3), then double-passed through a set of four lenses (L4-L7) with the retro-reflection occuring at the DM surface. Right: A 3D CAD rendering of the 'Imaka-Cassegrain layout showing the lenses and bottom end of the CFHT 3.6-m telescope. The large L4 and L5 lenses are roughly $1.2 \mathrm{~m} \mathrm{x}$ $0.8 \mathrm{~m}$ in size. 


\subsubsection{Cassegrain Focus Design}

John Pazder at HIA developed a design for the Cassegrain focus based on a Wynne-Dyson double-pass design that has a contiguous, on-axis one degree diameter field. The design is rather unique since several lenses are used double pass with the entrance beam passing to one side of lens center and the exit beam from the DM passing through the opposite side of the lenses (Figure 2). This design has not benefited from the level of optimization evident in the 'Imaka prime focus design, but still meets many of the design requirements. The design uses mostly refractive optics, except for the DM and a fold mirror. The $400 \mathrm{~mm}$ diameter DM has the advantage of being at the telescope pupil and slightly concave which facilitates testing. There is ample room for an ADC, filters, and wavefront sensors in the optical path. The full one degree beam diameter $(470 \mathrm{~mm})$ easily passes through the primary mirrors central hole $(680 \mathrm{~mm})$ with clearance. Three lenses are mounted either inside the primary mirror cell or in the primary mirrors central hole.

The design's key advantages are that the image quality is excellent across the one-degree diameter field of view and the pupil image is sharp (less than $2 \%$ pupil distortion in the worst case) with the DM positioned at the telescope pupil. The designs chief drawbacks are its very large size and thus its weight, several very large lenses, 22 air-glass interfaces without counting the ADC, filter or cryostat window, a refigured secondary mirror, and the loss of the Cassegrain Bonnette which will need to be removed, probably on a permanent basis.

While both optical designs meet the basic performance and functional requirements they differ significantly in all other means. As a result, we will need to down-select the optical design early in the Phase A study.

\subsection{GLAO}

The details of the GLAO design have not been a high-priority during the feasibility study with the exception of constraining the DM altitude conjugation and the allowable tilt of the DM with respect to the optical axis. In addition basic simulations for the order of the system (10x10 to 20x20 actuators) and the effects of guide asterisms were made. We see the engineering challenges for the next phase of the study to be: (1) the control algorithms and wavefront reconstruction and (2) the necessity for multiple deployable wavefront sensors. We note that while the acquisition system for the wavefront sensors could be simplified if the system used laser guide stars (for example multiple Rayleigh beacons), the additional complexities of deploying multiple lasers and its associated launch facility are viewed as incompatible with the CFHT model of operation. Given the large fields of view, the sky coverage with natural guide stars permits the use of natural stars for 6-8 guide stars.

The hardware for the individual components largely exists already. The individual wavefront sensors are similar to wavefront sensors operating in AO systems today. The deformable mirrors are larger than typical DMs and also have slightly curved (spherical) surfaces. At least one DM manufacturer feels these DMs are within their current capabilities. The real-time AO controller must process many subapertures and will benefit from a tomographic wavefront reconstructor. So while the system loop sampling rate to handle the ground-layer and dome seeing is expected to be lower than typical AO systems, the wavefront reconstruction may benefit from dedicated hardware wavefront reconstruction solutions such as those envisioned for the AO systems on extremely large telescopes such as TMT.

\subsection{OTCCDs}

Orthogonal transfer $\mathrm{CCDs}^{8}$ provide the means to shuffle charge between adjacent pixels at high transfer rates while continuing to collect charge. Operationally, bright stars within the field are identified and the pixels within the individual cell of pixels (typically 600x600 pixels) around these stars are readout at high sub-frame rates (e.g. $30 \mathrm{~Hz}$ ) to determine the real-time jitter errors. These guide star signals are then fed back to the rest of the detector cells to shuffle their charge appropriately. This provides a focal-plane solution to residual wavefront tip/tilt errors. Corrections for the atmospheric wavefront tilt are made over individual isokinetic patch regions of the sky (arcminutes in size) and the telescope tracking/jitter errors are also reduced. The maturity of OTCCDs is rapidly advancing with detectors in operation at the UH2.2m telescope on Mauna Kea and at the 3.5-meter WIYN telescope on Kitt Peak. In addition, the PanSTARRS ${ }^{9}$ and WIYN/One-Degree imager ${ }^{10}$ (ODI) projects have/are populating large focal planes with OTCCDs. 'Imaka is a natural progression of these projects and the baseline focal-plane array is very similar to the PanSTARRs-1 and ODI giga-pixel cameras. 
As with the GLAO components, the OTCCD and the camera/control electronics were not perceived as feasibility concerns since the PS1 ${ }^{11}$ and ODI cameras requirements are very similar to that needed for 'Imaka. Of note however for the 'Imaka OTCCDs are two outstanding issues. First, while the Optic ${ }^{12}$ and the QUOTA ${ }^{13}$ cameras have demonstrated the performance and gains of OTCCD for astronomical observations, charge shuffling for the very large arrays and fields of view (e.g. PS1 and ODI) and the optimal use of the guide signals and pixel control must be done. Since roughly half the fields at the North Galactic Pole (NGP) will not have enough bright, isolated stars to fully sample all the iso-kinetic patches (a few arcminutes in size) over one degree field of view, the optimal use of the available guide stars is a key question. In fact the sky coverage for full GLAO+OTCCD correction is driven by the need for sufficient OTCCD guide stars ( 150) rather than the GLAO correction. The second consideration for 'Imaka's choice of OTCCD sensors rests in the desired wavelength coverage. The "Imaka science case calls for a broad wavelength coverage but the current OTCCD detectors are either "thick" and optimized for red wavelengths (e.g. 0.45-1.1 microns) or "thin" and optimized for blue wavelengths $(0.35-0.9$ microns). Given that the resolutions achieved with the GLAO and OTCCD are best at the red wavelengths and that the ODI camera will be using the blue-sensitive detectors, we expect that 'Imaka will be optimized for the reddest wavelengths.

\section{SITE CHARACTERISTICS}

The 'Imaka concept works because the delivered image quality is degraded largely by optical turbulence within a volume close to the telescope and/or ground. The CFHT MegaCam image quality study ${ }^{4}$ and the Gemini ground-layer study ${ }^{3}$ show that this is indeed the case for CFHT on Mauna Kea. Salmon et al. ${ }^{4}$ deduce the relative contributions to the delivered image quality of MegaCam images due to a variety of sources and they find that the largest local degradations come from dome seeing ( 0.43 " at 0.5 microns) and the telescope static optical aberrations (0.33" at 0.5 microns). Using an optical turbulence profiler, Chun et al. ${ }^{3}$ found that the atmosphere above the summit of Mauna Kea has a very thin, 30-40 meter thick layer of optical turbulence just above the ground and very little optical turbulence elsewhere in the boundary layer (e.g. first kilometer). Finally, multiple studies ${ }^{2,3}$ have found that the free-atmosphere seeing above Mauna Kea is excellent (0.35-0.4" FWHM at 0.5 microns). All of these results suggest that GLAO on CFHT will provide a significant improvement to the delivered image quality of the facility over a very wide field of view.

For the feasibility study of 'Imaka we set out to (1) confirm the findings from Salmon et $\mathrm{al}^{4}$ and Chun et al. ${ }^{3}$ using an optical turbulence profiler on the CFHT telescope and (2) to quantify the distribution and spatial and temporal characteristics of the optical turbulence in and just outside the dome for input into the 'Imaka performance simulations/error budget. To do this we've begun a two-staged experiment. The first phase of the optical turbulence profiler (OTP1), now deployed at a bent-Cassegrain port on CFHT, consists of a single Shack-Hartmann wavefront sensor that measures the wavefront tilts over a one-meter off-axis portion of the CFHT primary.

OTP1 resides at a bent-Cassegrain port of the CFHT and is configured to be a 20x20 subaperture ShackHartmann wavefront sensor using a square 1 meter $\mathrm{x} 1$ meter portion of the CFHT primary mirror. It collects data, typically for 15 minutes, on nights when CFHT's Cassegrain science instrument (ESPANDONS) is being used. With a single star WFS an ambiguity remains on the altitude at which the turbulence arises. However, the phase power spectrum of the turbulence shows a bimodal distribution of temporal frequencies for the phase aberrations (Figure 3). We identify (but have not confirmed) the low temporal frequency power $(f \sim 0.1 \mathrm{~Hz})$ as arising within the dome and the high frequency power as the atmospheric component. We have some assurance that this is the case for at least a few nights when we ran an optical turbulence profiler outside the CFHT facility in addition to OTP1. For these nights we can identify distinct layers in the slope/phase temporal crosscorrelations. Two distinct layers are common to both experiments while an additional low temporal frequency component is seen only in the CFHT/OTP data. specific to CFHT.

For now, making a somewhat arbitrary cut at $1 \mathrm{~Hz}$ in the phase power spectra to to distinguish "dome seeing" and "atmospheric seeing", we find that the optical turbulence arising within the dome is significant and often the dominant contribution. On average we find over the 56 nights sampled to date that the low- temporal frequency component $(f<1 \mathrm{~Hz})$ is equal to or even slightly larger than the high- frequency component $(f>1 \mathrm{~Hz})$. All 

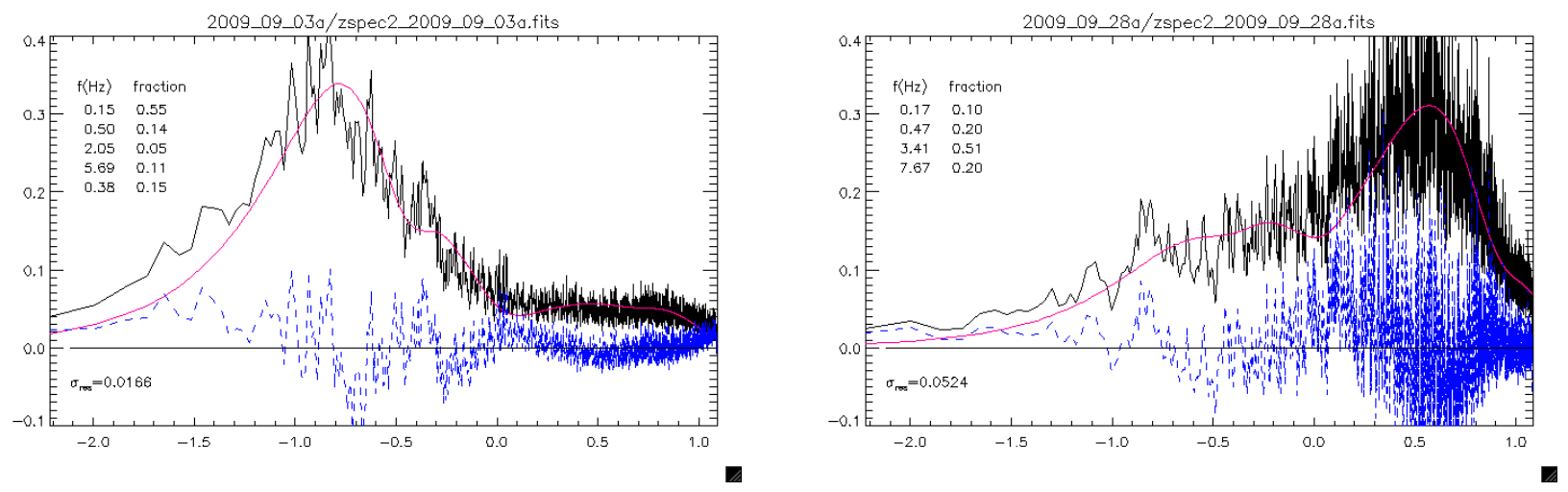

Figure 3. Two examples of the power spectral density of Zernikes $4 / 5$ from OTP1. The data are shown in black while five-layer fits are shown in red. The data is shown in $f^{*} p s d(f)$ versus $\log (f)$ as in Roddier et al. (1995) where the area under the curves are proportional to the total variance in the layer. The blue curve shows the residual after the model fit. The two nights show dramatically different distributions in frequency space. The 2009-09-03 data appears to be nearly entirely due to dome seeing while the atmospheric seeing dominates in the 2009-09-28 data set. The normal distribution is a more balanced mixture of the two.

studies (here with OTP, Salmon et al., ${ }^{4}$ and Racine et al. $(1991)^{1}$ find similar fractions of contributions from the dome $(\sim 0.45 ")$ and atmosphere $(\sim 0.45 ")$.

In the second phase of OTP, we will deploy a true profiler (e.g. SLODAR/LOLAS) at the CFHT bentCassegrain port. These true profilers will obtain the turbulence altitudes directly and will help to remove any final ambiguities in the origin of the turbulence.

Finally, we note that there are now a number of additional profilers and seeing monitors on the summit. There are two seeing monitors on the summit of Mauna Kea: the CFHT DIMM in the slit of the CFHT enclosure and the summit facility Mauna Kea Atmospheric Monitor (MKAM). MKAM is a low-resolution optical turbulence profiler and seeing monitor (MASS/DIMM) sited at the location of the old CFHT/Gemini weather tower next to CFHT. This facility now provides the strength and distribution of atmospheric seeing on a nightly basis. Over the course of the first few months of data with MKAM the median MASS seeing (essentially free-atmosphere seeing as it is measured from $500 \mathrm{~m}$ and above) was measured to be 0.3 ". This is in excellent agreement with past studies at Mauna Kea. In addition, a high-resolution optical profiler (Lunar SHABAR) is being deployed in a campaign mode on the summit by Paul Hickson/Thomas Pfrommer (UBC) and will provide very high resolution profiles from inside the dome and at the MKAM site.

\section{PERFORMANCE SIMULATIONS}

In this section we present the highlights of the 'Imaka performance simulations developed during the feasibility study. The details of the simulations, and importantly the development of the effects of tilting the DM with respect to the optical axis and the conjugation of the DM, are described in these proceedings by Lai et al..

A new simulation code specifically for 'Imaka that incorporates a numbers of unique features was developed. The code includes a model of the atmospheric turbulence based on the Gemini Mauna Kea Ground-layer study, the effects of misconjugating and tilting a DM with respect to the optical axis, and the effects of the combined correction from a GLAO system and the tip/tilt correction from an orthogonal-transfer CCD camera. The code (instantGLAO) was developed by Olivier Lai and has been extensively cross-compared for a number of case studies with the yao and simul codes (Francois Rigaut), the LAOS code (Brent Ellerbroek/Luc Gilles), and PAOLA (Laurent Jollisaint). In addition, PAOLA has been modified to be used with 'Imaka and is now being used to develop a detailed baseline for the GLAO system. With instantGLAO we quantified the phase errors due to the tilt of the DM with respect to the optical axis and the optical conjugation of the DM with respect to the optical turbulence. These errors place strong constraints on the 'Imaka optical design and drove the optical design away from an adaptive secondary due to its misconjugation altitude. This is a major change from the original work. ${ }^{7}$ 


\subsection{Input to the simulations}

The delivered performance of the GLAO system and the OTCCD will depend on the distribution in altitude and time and the strength of optical turbulence within the atmosphere and dome. The major components of the image degradation at CFHT are the atmosphere, the dome seeing, and the telescope primary mirror figure. ${ }^{4}$ For the atmospheric contribution, we used the $25 \%, 50 \%$, and $75 \%$-tile values of the ground-layer and freeatmosphere strengths ${ }^{3}$. These two uncorrelated regions give rise to nine different atmospheric conditions. The turbulence profile was taken to be the median GL distribution found in Chun et al. ${ }^{3}$ The median dome seeing and the primary mirror figure errors are taken from Salmon et al. ${ }^{4}$ In addition the optical design aberrations are included in the simulations. The CFHT dome seeing ${ }^{4}$ results in a significant degradation to the delivered performance of 'Imaka and the effort to reduce the locally generated seeing by venting the dome is a key element to the 'Imaka development. Note however that while dome venting is being actively pursued, we have pessimistically maintained the full dome seeing in the 'Imaka performance simulations. Table 2 shows the nine cases considered in terms of the total integrated seeing.

Table 2. The simulation input integrated seeing conditions for the nine combinations of $25 \%, 50 \%$, and $75 \%$-tile ground layer (GL) and free atmosphere (FA) plus the median dome seeing (0.45”) and static primary mirror figure. The atmosphere-only integrated seeing values are given for reference in parentheses.

\begin{tabular}{|l|c|c|c|}
\hline & Good FA (0.31") & Median FA (0.42") & Bad FA (0.55") \\
\hline Good GL (0.35") & $0.715 "(0.50 ")$ & $0.785 "(0.59 ")$ & $0.877 "(0.70 ")$ \\
\hline Median GL (0.47") & $0.794 "(0.60 ")$ & $0.860 "(0.68 ")$ & $0.947 "(0.78 ")$ \\
\hline Bad GL (0.64") & $0.921 "(0.75 ")$ & $0.981 "(0.82 ")$ & $1.060 "(0.91 ")$ \\
\hline
\end{tabular}

The delivered performance of 'Imaka will also depend on the spatial distribution of guide stars within the field of view. The probability of finding at least $n$ stars brighter than magnitude $m$ within the field of view is given by:

$$
P(m,>n)=1-\sum_{i=1}^{n}(\mu(m))^{i} \frac{e^{-\mu(m)}}{i !}
$$

where $\mu(m)$ is the mean star density of magnitude $\mathrm{m}$ from the Besanon model of star counts for a field of view of 1 degree $(\mathrm{r}=30)$. The large field of 'Imaka works to its advantage as it is found that there is a $>95 \%$ probability of finding 6-8 stars of $m_{R}<12$ within one square degree at the North Galactic Pole. The sky coverage for full correction will be largely driven by the requirement of tip/tilt guide stars for the OTCCD correction and is estimated to be around 60-70\% at the NGP. The actual implementation of the wavefront sensors (placement of wavefront sensors with respect to the science filters, arms interference, etc.) will work to reduce this and this will be explored in the design phase.

Simulations show that for more than four guide stars, the GLAO correction is largely unchanged. To give a feeling for the dependence (or lack thereof) of the delivered performance on the constellation of GLAO and OTCCD guide stars, we chose to position the guide stars in the simulation as found in a representative field. We used the COSMOS field (http://cosmos.astro.caltech.edu/) due to its importance as a field for extra-galactic studies and its modest galactic latitude $(\approx 40$ degrees). For this field we found 8 guide stars for GLAO and over 130 guide stars for OTCCD correction within the 'Imaka field of view which proved to be sufficient for good correction over the full field. All of the following results use this 'COSMOS' field asterisms in the simulations.

\subsection{Summary of results}

\subsubsection{End-to-End performance}

The end-to-end numbers are given in Table 3. They are very similar to the 'end-to-end' numbers we quoted in the original study. ${ }^{7}$ Though we now include more detrimental effects (dome seeing, mirror figure, and the tilt of the DM), we also moved away from a concept based on an adaptive secondary. The gain by conjugating the deformable mirror correctly (as opposed to the -43 meter conjugation of the adaptive secondary) and the inclusion of the dome and mirror figure errors largely balance each other. At the bluest wavelengths the increase 
in the total seeing included in the simulations degrades the performance significantly and will benefit greatly from the dome venting program. We are also now including Y-band (1.0 microns) in the simulation runs as it was identified as an important bandpass for the science cases.

Table 3. 'Imaka End-to-End performance for the 'Imaka-Cassegrain design in median(GL)/median(FA) atmospheric conditions including dome seeing and the primary mirror figure. The table shows the $25 \%, 50 \%$, and $75 \%$-tile FWHM over the one deg diameter FOV for five wavelengths of interest with GLAO-only correction and full GLAO+OTCCD correction. For reference, the input integrated seeing was $0.86 "$ at $500 \mathrm{~nm}$.

\begin{tabular}{|c|c|c|}
\hline $\begin{array}{c}\text { Science Wavelength } \\
\text { (filter) }\end{array}$ & $\begin{array}{c}\text { GLAO-only } \\
\text { FWHM (arcsec) }\end{array}$ & $\begin{array}{c}\text { GLAO+Full OTCCD } \\
\text { FWHM }(\operatorname{arcsec})\end{array}$ \\
\hline 0.45 microns (b) & $0.47,0.49,0.50 "$ & $0.39,0.40,0.42 "$ \\
0.5 microns (g) & $0.44,0.46,0.48 ”$ & $0.36,0.38,0.39 "$ \\
0.7 microns (r,i) & $0.38,0.40,0.41 "$ & $0.29,0.31,0.32 "$ \\
0.9 microns (i,z) & $0.35,0.36,0.38 "$ & $0.25,0.27,0.29 "$ \\
1.0 microns (Y) & $0.34,0.35,0.37 ”$ & $0.24,0.25,0.28 ”$ \\
\hline
\end{tabular}

\subsubsection{How does the image quality vary across the field?}

The performance, as measured by the FWHM, is remarkably uniform over the one degree field of view. For the COSMOS field, eight GLAO guide stars were found near the periphery of the field and over 130 OTCCD guide stars were found within the one-degree diameter field of view. Figure 3 below shows a color-coding of the FWHM derived from an array of 60x60 PSFs (PSFs sampled every one arcminute) across the field. Positions of the eight GLAO guide stars are indicated by yellow stars while the OTCCD guide star positions are indicated by crosses. With an OTCCD focal-plane populated with OTCCDs the detector real estate immediately surrounding an OTCCD guide star is used for sensing the tip/tilt correction. As such, these areas are not accessible for science. The area lost to the OTCCD guide stars is about 1 square arcminute per guide star so in the case of the COSMOS field, this amounts to less than $5 \%$ of the field. In addition, the areas surrounding the GLAO guide stars will be vignetted by the GLAO WFS probe arms. These areas are also not accessible for science. Finally, the area vignetted by the WFS patrol arms will depend on the design of the WFS arms but the intent is to minimize this obstruction.

Given that in some fields there will not be enough guide stars for full OTCCD correction over the field of view, we list the performance of both the GLAO-only (an extreme of no OTCCD guide stars) and full GLAO+OTCCD cases. The OTCCD correction amounts to an improvement of about 0.1" in FWHM at all wavelengths. Comparing the GLAO-only and the GLAO+OTCCD PSF FWHM across the field seems to point to the OTCCD contributing in two ways. First it removes the tip and tilt from the upper atmospheric layers. Second, it smooths out the GLAO performance by correcting for tilt anisoplanatism within the GLAO correction. We note that the OTCCD performance across the field depends on the altitude of the free-atmosphere layers and on how we combine information from multiple OTCCD guide stars to generate the correction signal. Here a simple averaging of the wavefronts is used (e.g. not tomographic). More advanced techniques will be pursued in the Phase A study. We will also be using the MKAM MASS/DIMM seeing monitor to develop a better turbulence model of for the free atmosphere.

\subsubsection{Probability of obtain a particular image quality}

For a particular observing time and location in the sky, the seeing and the number of appropriate guide stars will vary. For the range of atmospheric seeing considered (e.g. the quartile strengths of the ground-layer and freeatmosphere), we can derive rough probabilities that a particular combination will occur given that the turbulence in the ground-layer and free-atmosphere are uncorrelated. This gives rise to nine different seeing conditions used (outlined above in section 4). Figure 5 shows the median delivered image quality in the 'Imaka field of view at 0.7 microns given the range in atmospheric conditions, the median dome seeing value, the primary mirror figure error, and the camera optical design aberrations. 

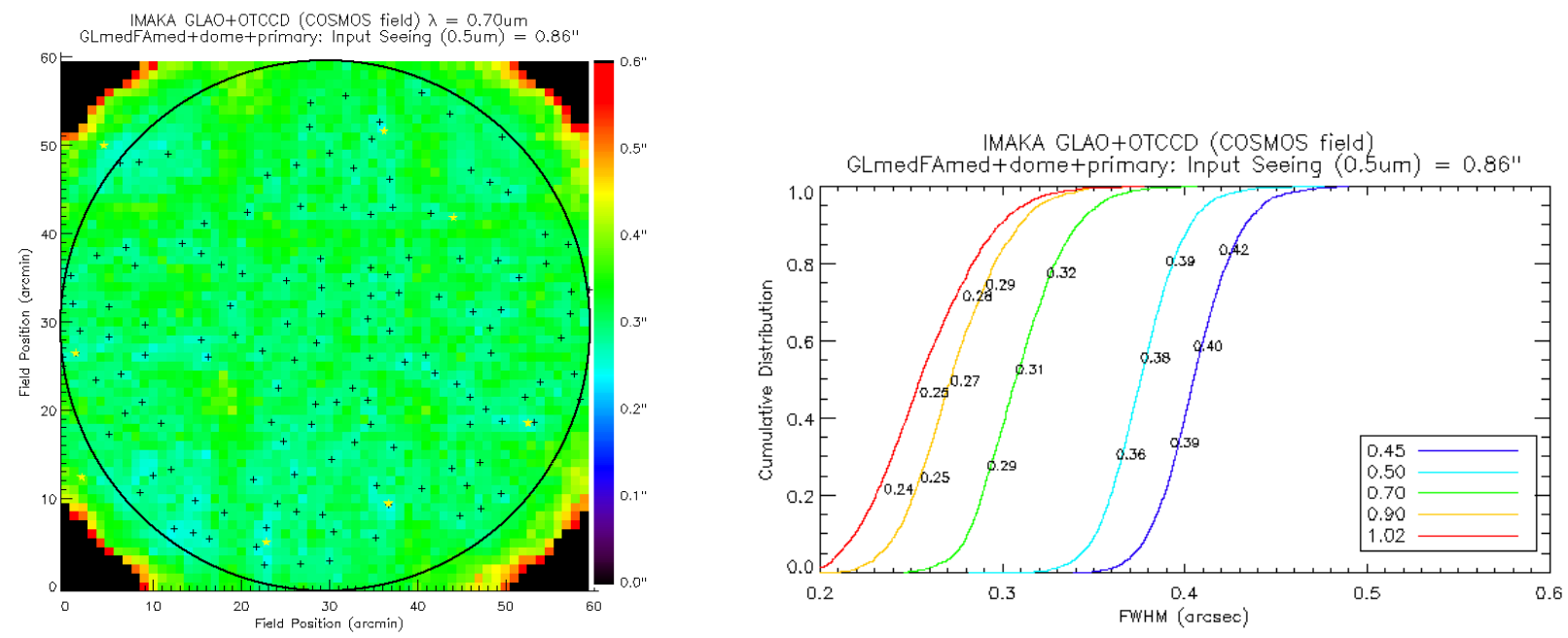

Figure 4. Left: The distribution of FWHM across the one-square degree field at an imaging wavelength of 0.7 microns for median ground-layer and median free-atmosphere strengths. The one degree diameter field of the 'Imaka-Cassegrain design is shown as well as the positions of the GLAO guide stars (yellow stars) and the OTCCD guide stars (crosses). The sharp rise in the FWHM in the corners of the field are nearly entirely due to the optical design aberrations that degrades quickly outside the one-degree diameter. Right: The cumulative probability distribution of FWHM within the one-degree diameter field. The distribution of FWHM for the five wavelengths are shown. For each wavelength the $25 \%$, $50 \%$, and $75 \%$-tile FWHM in the field are noted next to the curve.

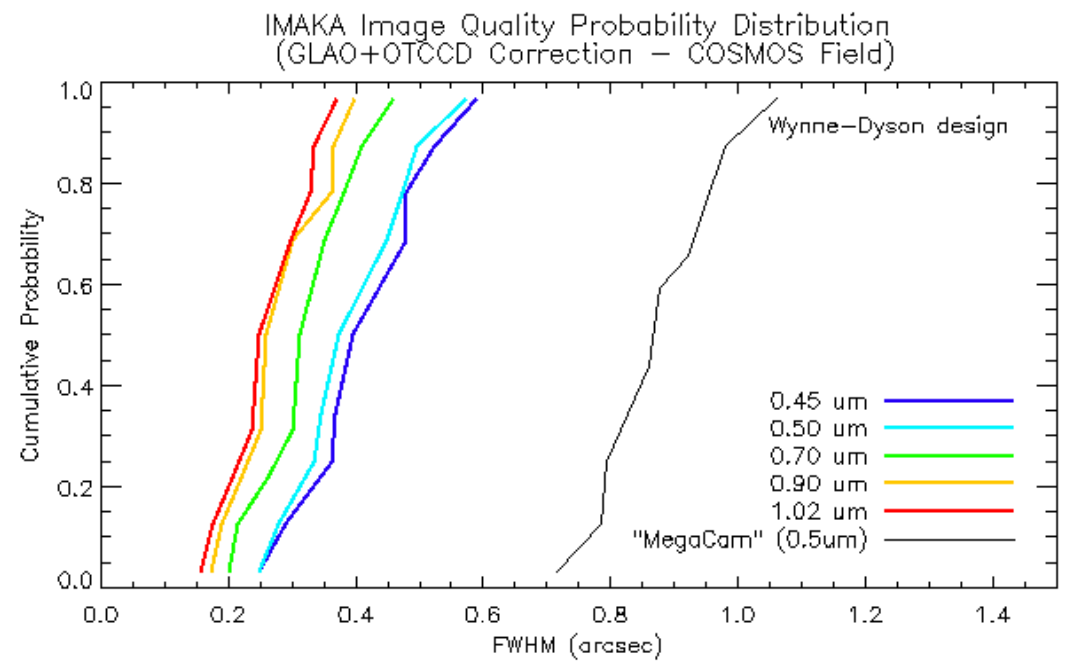

Figure 5. Cumulative probability distribution function for 'Imaka for the adopted set of input seeing conditions. The curves show the probability that the median FWHM within the field will be less than or equal to a value for each of the five science wavelengths in question. The thin black line gives a reference for the input image quality (including the atmospheric seeing, the optical design, dome seeing, and mirror figure) for the 'Imaka Cassegrain Wynne-Dyson design at a wavelength of 0.5 microns. It should be comparable to the delivered image quality obtained with the current CFHT/MegaCam imager though the best conditions are pessimistic while the worst conditions are optimistic when compared to the actual image quality statistics for MegaCam. This is largely due to the fact that we have considered a single (median) value for the dome seeing. 


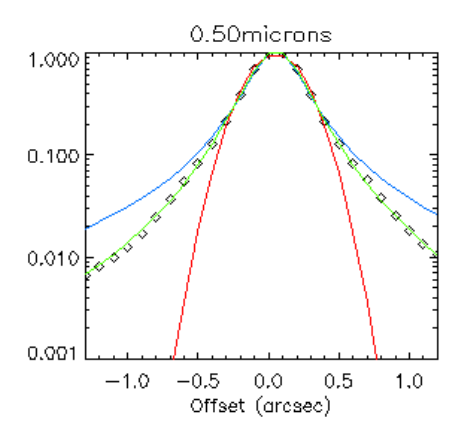

IMAKA PSF Shapes (GLAO+OTCCD-COSMOS)
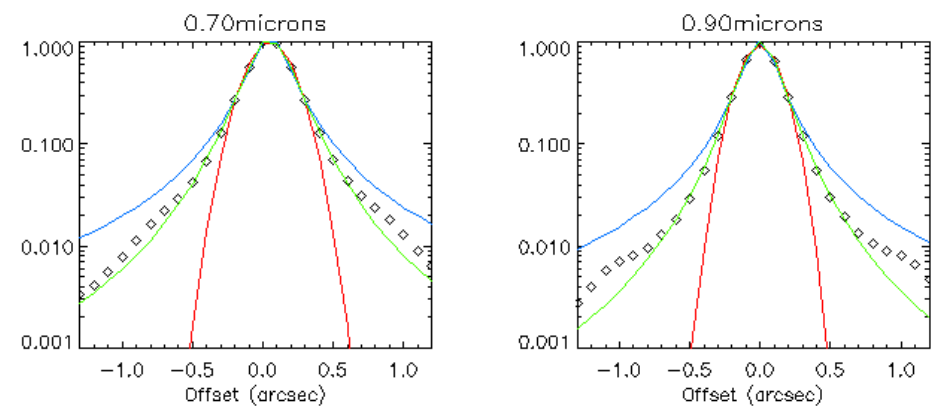

Fits: Gaussian, Lorentzian, Moffat

Figure 6. The shape of the 'Imaka PSFs is shown by the symbols. These cuts were made on the average PSF within the field for the median(GL)/median(FA) case at wavelengths of $0.5,0.7$, and 0.9 microns. Three functional fits are also shown Gaussian (red), Moffat (green), and Lorentzian (blue).

\subsubsection{Shape of the PSF}

The 'Imaka PSF is dominated by the residual free-atmosphere seeing and as such we expect the PSF will be more like a typical seeing-limited image than the canonical PSF of a classical AO system. Indeed, at the bluer wavelengths the shape is well described by a Moffat profile (Figure 6). At the redder wavelengths the residual wavefront aberrations are small enough that the PSF deviates from this shape and we begin to see a halo and core to the PSF with deviations from the Moffat profile of a few percent in intensity in the wings of the PSF. The figure below shows a cut of the average PSF within the one-degree diameter field of view of 'Imaka for the COSMOS simulations. Only the wavelengths $0.5,0.7$, and 0.9 microns are shown. Each average PSF is fit with a Gaussian, Lorentzian, and Moffat profile. The Gaussian and Lorentzian profiles are never good fits. The Moffat profile is a good fit for the bluer wavelengths (namely where the phase variance is still large) but starts to deviate from the PSF at the longer wavelengths.

\section{FUTURE WORK}

We believe we have addressed within this study the basic feasibility and gains of a wide-field GLAO system plus OTCCDs at CFHT. Given the optical turbulence distribution near the ground on Mauna Kea a GLAO system is very effective. The combination of GLAO+OTCCD brings high resolution images across one-deg fields with high sky coverage.

Although the individual concepts of GLAO and OTCCDs are understood, the implementation here is innovative and there are subtle issues that need to be kept in mind as the instrumental matures. Some pending issues are due to lack of sufficient data or lack of time to explore them in detail. They will need to be answered in any further studies.

- CN2(h)dh profile: To this point we have used the Ground layer profile that was measured from the roof of the UH 88 " telescope. This had just enough resolution $(d h \sim 15 m)$ to discern two thin layers near the ground. The OTP experiment has provided some statistics of the relative occurrence of dome, ground layer and free atmosphere seeing, but without probing their vertical extent. More statistics and confirmation of the conclusion of the MKGL study are needed to validate the entire concept. The path to achieve this is to be able to sense the turbulence at higher resolution through the telescope and dome itself. This is the goal of the OTPv2 experiment. The very high vertical resolution profiles can be fed to the simulations to improve the realistic performance of GLAO. In addition, if multiple cameras/stars can be used over a large enough field, then an on-sky validation of 'Imaka can be made using the wavefront sensor telemetry. ${ }^{14}$

- Missing error terms in GLAO budget: The current error budget accounts for the dominant error terms. A full system error budget will need to include error terms associated with wavefront sensing, temporal lag 
(which we can obtain from PAOLA), and reconstruction error. While these terms are all small compared to the free atmosphere seeing and residual error, care has to be taken that they do not add excessively. Finally, there are some error terms related to real world implementation that cannot easily be simulated ab initio, but can be included as they arise to constantly monitor the expected performance during development. These include aberrations introduced by optical elements, alignment errors, or what kind of dome seeing to expect after the dome will have been vented.

- OTCCD performance: The performance of OTCCD arrays is still subject to debate especially in an operational context over these large fields of view. We have currently implemented a simple model of nearest neighbor measurement and integer pixel correction; we also neglect the bandwidth error which might be large for high altitude Taylor flows and $100 \mathrm{~Hz}$ sampling rate. Fortunately, the PanSTARRS1 and ODI teams should address these issues in the coming year. Another area of concern regarding the simulations is the actual strength and distribution of the free-atmosphere turbulence: to prevent wrap-around, the altitude of the highest phase screen is set to $3000 \mathrm{~m}$, which is enough for GLAO purposes (the guide star beams are completely decorrelated above $300 \mathrm{~m}$ ), although it is more likely concentrated between 6000 to $9000 \mathrm{~m}$ above the telescope. This means that the isokinetic angle may in fact be smaller than currently expected from our simulations. The MKAM MASS/DIMM will provide statistically valuable data as input to the model.

- Zenith/thickness of GL dependence. So far all the simulations assume that the thickness of the optical turbulence is the same as the vertical profile; this implies that they are valid at zenith. When pointing over at a zenith angle $\alpha$, the effective thickness of the layer is multiplied by a factor $1 / \cos \alpha$. While 'Imaka is conceived as a near zenith instrument anyway due to the zenith angle dependence of $r_{0}$, the effect of observing $30^{\circ}$ from zenith will be to effectively reduce the size of the GLAO corrected field by a factor 1.15 in the linear gray zone approximation. This effect needs to be simulated in greater detail to understand the limits of GLAO off-zenith.

\section{ACKNOWLEDGMENTS}

The feasibility study was funded by the Canada-France-Hawaii Telescope Corporation.

\section{REFERENCES}

[1] Racine, R., Salmon, D., Cowley, D., and Sovka, J., "Mirror, dome, and natural seeing at cfht," Astronomical Society of the Pacific 103, 1020 (Sep 1991).

[2] Schöck, M., Els, S., Riddle, R., Skidmore, W., Travouillon, T., Blum, R., Bustos, E., Chanan, G., Djorgovski, S. G., Gillett, P., Gregory, B., Nelson, J., Otárola, A., Seguel, J., Vasquez, J., Walker, A., Walker, D., and Wang, L., "Thirty meter telescope site testing i: Overview," Publications of the Astronomical Society of the Pacific 121, 384 (Apr 2009).

[3] Chun, M., Wilson, R., Avila, R., Butterley, T., Aviles, J.-L., Wier, D., and Benigni, S., "Mauna kea groundlayer characterization campaign," Monthly Notices of the Royal Astronomical Society 394, 1121 (Apr 2009).

[4] Salmon, D., Cuillandre, J.-C., Barrick, G., Thomas, J., Ho, K., Matsushige, G., Benedict, T., and Racine, R., "Cfht image quality and the observing environment," Publications of the Astronomical Society of the Pacific 121, 905 (Aug 2009).

[5] Bec, M., Rigaut, F. J., Galvez, R., Arriagada, G., Boccas, M., Gausachs, G., Gratadour, D., James, E., Rojas, R., Rogers, R., Sheehan, M. P., Trancho, G., and Vucina, T., "The gemini mcao bench: system overview and lab integration," Adaptive Optics Systems. Edited by Hubin 7015, 173 (Jul 2008).

[6] Mouhcine, M., Ibata, R., and Rejkuba, M., "A panoramic view of the milky way analog ngc 891," The Astrophysical Journal Letters 714, L12 (May 2010).

[7] Lai, O., Chun, M., Cuillandre, J. C., Carlberg, R., Richer, H., Andersen, D., Pazder, J., Tonry, J., Doyon, R., Thibault, S., Dunlop, J., Pritchet, C., Véran, J. P., Ftaclas, C., Onaka, P., Hodapp, K. W., McLaren, R. A., Bertin, E., Mellier, Y., Astier, P., and Pain, R., "Imaka: imaging from mauna kea with an atmosphere corrected 1 square degree optical imager," Adaptive Optics Systems. Edited by Hubin 7015, 121 (Jul 2008). 
[8] Tonry, J., Burke, B. E., and Schechter, P. L., "The orthogonal transfer ccd," Publications of the Astronomical Society of the Pacific 109, 1154 (Oct 1997).

[9] Kaiser, N., Aussel, H., Burke, B. E., Boesgaard, H., Chambers, K., Chun, M. R., Heasley, J. N., Hodapp, K.-W., Hunt, B., Jedicke, R., Jewitt, D., Kudritzki, R., Luppino, G. A., Maberry, M., Magnier, E., Monet, D. G., Onaka, P. M., Pickles, A. J., Rhoads, P. H. H., Simon, T., Szalay, A., Szapudi, I., Tholen, D. J., Tonry, J. L., Waterson, M., and Wick, J., "Pan-starrs: A large synoptic survey telescope array," Survey and Other Telescope Technologies and Discoveries. Edited by Tyson 4836, 154 (Dec 2002).

[10] Jacoby, G. H., Tonry, J. L., Burke, B. E., Claver, C. F., Starr, B. M., Saha, A., Luppino, G. A., and Harmer, C. F. W., "Wiyn one degree imager (odi)," Survey and Other Telescope Technologies and Discoveries. Edited by Tyson 4836, 217 (Dec 2002).

[11] Onaka, P., Tonry, J. L., Isani, S., Lee, A., Uyeshiro, R., Rae, C., Robertson, L., and Ching, G., "The panstarrs gigapixel camera \#1 and stargrasp controller results and performance," Ground-based and Airborne Instrumentation for Astronomy II. Edited by McLean 7014, 12 (Aug 2008).

[12] Tonry, J. L., Burke, B. E., Luppino, G., and Kaiser, N., "The orthogonal parallel imaging transfer camera," Scientific Detectors for Astronomy 300, 385 (Jan 2004).

[13] Jacoby, G., Howell, S., Harbeck, D., and Sawyer, D., "Quota - an advanced mosaic imager," American Astronomical Society 213, 428 (Jan 2009).

[14] Baranec, C., Lloyd-Hart, M., and Milton, N. M., "Ground-layer wave front reconstruction from multiple natural guide stars," The Astrophysical Journal 661, 1332 (Jun 2007). 